Vulnerabilidades urbanas en los países andinos

(Bolivia, Ecuador, Perú)

\title{
Vulnérabilités urbaines dans les pays andins. Introduction générale
}

Robert D'Ercole, Sébastien Hardy, Pascale Metzger et Jérémy Robert

\section{(2) OpenEdition}

Journals

Édition électronique

URL : http://journals.openedition.org/bifea/2227

DOI : $10.4000 /$ bifea.2227

ISSN : 2076-5827

Éditeur

Institut Français d'Études Andines

Édition imprimée

Date de publication : 1 décembre 2009

Pagination : 411-420

ISSN : 0303-7495

Référence électronique

Robert D’Ercole, Sébastien Hardy, Pascale Metzger et Jérémy Robert, « Vulnérabilités urbaines dans les pays andins. Introduction générale », Bulletin de l'Institut français d'études andines [En ligne], 38 (3) | 2009, mis en ligne le 01 juin 2010, consulté le 19 novembre 2020. URL : http:// journals.openedition.org/bifea/2227 ; DOI : https://doi.org/10.4000/bifea.2227

\section{(c) $($ ) $\odot \ominus$}

Les contenus du Bulletin de l'Institut français d'études andines sont mis à disposition selon les termes de la licence Creative Commons Attribution - Pas d'Utilisation Commerciale - Pas de Modification 4.0 International. 


\title{
Vulnérabilités urbaines dans les pays andins Introduction générale
}

\author{
Robert D'Ercole* \\ Sébastien Hardy** \\ Pascale Metzger* \\ Jérémy Robert***
}

\section{VULNÉRABILITÉS URBAINES}

Ce numéro thématique du Bulletin de I'Institut Français d'Études Andines est une contribution à la connaissance de la vulnérabilité urbaine dans trois pays andins : la Bolivie, l'Équateur et le Pérou. Par vulnérabilité urbaine nous entendons la difficulté des villes à anticiper et à faire face à des phénomènes physiques ou anthropiques (séismes, inondations, glissements de terrain, incendies, explosions, pollutions, etc.) susceptibles de causer des dommages, à leur résister et à récupérer après leur survenue (Blaikie et al., 1994). La vulnérabilité urbaine est aussi la capacité des villes et de leurs habitants à engendrer les conditions de la survenue de ces phénomènes ou du moins à en aggraver les effets (D’Ercole et al., 2009).

En raison d'une urbanisation croissante souvent mal contrôlée et d'un contexte géodynamique et climatique défavorable, les trois pays andins sont confrontés à un grand nombre de risques. Ces derniers se convertissent fréquemment en

* Institut de Recherche pour le Développement (IRD), UR 029, programme Pacivur, Calle Teruel 357, Miraflores, Casilla 18-1209, Lima 18, Perú. E-mails:

robert.dercole@ird.fr ; pascale.metzger@ird.fr

** Institut de Recherche pour le Développement (IRD), UR 029, programme Pacivur, Av. Hernando Siles 5290 esq. calle 7, CP 9214 Obrajes, La Paz, Bolivia. E-mail: sebastien.hardy@ird.fr

*** Institut Francais d'Études Andines (IFEA, UMIFRE 17, CNRS-MAEE)-Université de Savoie, CISM, Laboratoire EDYTEM (CNRS). E-mail: Jeremy.Robert@univ-savoie.fr 
catastrophes qui affectent durement la population, les infrastructures, les biens publics et privés, l'économie de ces pays et par conséquent, les conditions de leur développement. Les territoires nationaux sont concernés dans leur ensemble, mais les milieux urbains apparaissent de plus en plus touchés par ces catastrophes. En effet, la population totale des trois pays s'élève à 50 millions d'habitants, dont plus de $70 \%$ sont des urbains1. Le rythme de croissance de la population urbaine a, dans l'ensemble, connu une légère décélération durant les dernières décennies. La population urbaine continue cependant à croître, ce qui se traduit par un étalement et une densification concomitants des espaces urbains, en dehors de toute considération des risques et des vulnérabilités.

La vulnérabilité urbaine des pays andins s'insère dans un cadre plus général. D'ici 2030, la population urbaine mondiale s'élèvera à 5 milliards d'habitants et les villes des pays en développement abriteront plus de $80 \%$ de la population urbaine de la planète (UNFPA2, 2007). Ces projections s'appuient sur des hypothèses de taux de croissance urbaine soutenus en Amérique latine, forts en Asie et explosifs en Afrique. Par ailleurs, cette population urbaine est très concentrée dans l'espace, occupant à peine $1 \%$ de la superficie terrestre (Pelling, 2003). Ces données quantitatives sont souvent avancées pour expliquer la vulnérabilité croissante des villes, notamment des villes du Sud, face aux catastrophes d'origine naturelle et/ ou anthropique, et pour rendre compte du fait que la liste des villes frappées par une catastrophe majeure ne cesse de s'allonger et de se diversifier (Dubois-Maury \& Chaline, 2002). Ces données ne sont cependant acceptables qu'associées aux transformations qualitatives des agglomérations urbaines qui contribuent de manière considérable à leur vulnérabilité.

Les chercheurs soulignent, d'une part, la complexité croissante des villes (Pigeon, 1996 ; Pigeon 2007) : dépendance toujours plus forte de l'urbain vis-à-vis des grands réseaux techniques (Dubois-Maury \& Chaline, 2002), multiplication et imbrication toujours plus dense de ces réseaux, des flux de pouvoirs, d'énergie ou d'information (Pelling, 2003). Ils insistent, d'autre part, sur la rapidité de cette dynamique d'urbanisation qui produit des effets négatifs observés sous divers angles (D’Ercole, 1994 ; Lavell, 1999 ; Pelling, 2003) : dégradations environnementales (artificialisation des sols, surexploitation des ressources naturelles, pollutions, etc.) ; modification des sites urbains, étalement et occupation toujours plus grande d'espaces à risques (terrains en pente, lit majeur des cours d'eau, etc.) ; systèmes de construction souvent inappropriés ; absence de contrôles ; absence ou mauvaise qualité des infrastructures et services urbains ; législateurs et planificateurs qui peinent à suivre ces transformations, etc.

Les conditions dans lesquelles les villes des pays du sud se complexifient et se transforment ne peuvent qu'intensifier les vulnérabilités. Ces dernières relèvent

18274325 habitants et 62,4 \% de population urbaine en Bolivie (recensement INE, 2001) ; 14005449 et 65,7 \% en Équateur (projection INEC, 2009) ; 27412157 et 75,9\% au Pérou (recensement INEI, 2007).

2 United Nations Fund for Population Activities (Fonds des Nations Unies pour la population). 
de la pauvretés, de l'exclusion sociale, de la ségrégation spatiale, d'une histoire jalonnée d'erreurs, d'intérêts particuliers et de laisser-faire en matière de gestion et de planification urbaine, ou encore de systèmes de gouvernance pour lesquels la question des risques ne constitue pas une priorité. Ces conditions de l'urbanisation dans les pays du sud relèvent également de transformations économiques et sociales issues d'une vision universaliste du développement qui, par définition, s'affranchit des conditions locales qu'elles soient physiques, sociales ou culturelles. Dans les pays du sud, une bonne part de l'espace urbain échappe à l'aménagement au sens classique. La ville présente deux visages : la ville moderne et aménagée et la ville auto-construite et non équipée ; la ville légale et la ville illégale (D’Ercole et al., 2009).

À ces conditions de grande fragilité urbaine, issue des modalités même de la production urbaine et du développement, se greffe l'exposition aux aléas d'origine naturelle et anthropique. En 1995, on considérait que plus de $40 \%$ de la population urbaine des pays en développement était exposée à des aléas d'origine naturelle (D'Ercole \& Thouret, 1995). Pour sa part, Chester (2002) souligne que 86 des 100 plus grandes villes des pays du sud sont exposées à des aléas destructeurs (séismes, tsunamis, inondations, éruptions volcaniques, etc.). Les conséquences de cette exposition et des conditions de développement urbain des pays du sud sont extrêmement préjudiciables. Comme le signalait déjà Renaud Vié-le-Sage en 1989, plus de $90 \%$ des victimes des catastrophes naturelles vivent dans les pays en développement et chaque catastrophe y fait, en moyenne, 30 fois plus de victimes que dans les pays au niveau de vie le plus élevé4. La liste des catastrophes urbaines étayant ce propos est ainsi particulièrement longue et s'allonge inexorablement (tableau 1). Les catastrophes urbaines citées en exemple, pour la plupart liées à des séismes, mais aussi à des ouragans ou des éruptions volcaniques, se sont soldées par la mort de milliers, voire de dizaines de milliers de personnes.

Dans les trois pays andins étudiés dans ce volume, il faut remonter à plusieurs décennies pour trouver des catastrophes similaires. En Équateur, la ville de Pelileo fut détruite par un séisme en 1949, causant la mort de plus de 6000 personnes. Plus anciennement, d'autres villes ont été dévastées par des séismes comme Riobamba en 1797 (6 000 à 20000 morts selon les estimations). La ville de Latacunga fut pour sa part détruite à plusieurs reprises par les lahars (coulées de boues et de débris) produits par les éruptions du volcan Cotopaxi $(1742,1768,1877)$.

Au Pérou, un séisme accompagné d'un tsunami détruisit Lima et Callao en 1746 (plus de 3000 victimes). Le séisme de 1970 (70 000 morts) est resté ancré dans les mémoires. Il a notamment dévasté la ville de Huaraz et la localité de Yungay, cette dernière ayant été atteinte par une coulée de boue provoquée par l'effondrement

3 « Ce sont les pauvres qui alimenteront dans une grande mesure la croissance urbaine à venir » (UNFPA, 2007).

4 Dans le même ordre d'idées, Albouy indique que les victimes des catastrophes naturelles proviennent à $75 \%$ des pays les plus pauvres, à $23 \%$ des pays à revenus intermédiaires et à $2 \%$ des pays de I'OCDE (Organisation de Coopération et de Développement Economiques) (Albouy, 2002 : 40). 
Tableau 1 - Exemples de catastrophes urbaines à l'échelle mondiale

\begin{tabular}{|c|c|c|c|c|}
\hline Ville & Pays & Année & $\begin{array}{c}\text { Nature de } \\
\text { l'évènement }\end{array}$ & $\begin{array}{l}\text { Estimation } \\
\text { du nombre } \\
\text { de morts }\end{array}$ \\
\hline Agadir & Maroc & 1960 & séisme & 120001 \\
\hline $\begin{array}{l}\text { Huaraz, Yungay, } \\
\text { Chimbote }\end{array}$ & Pérou & 1970 & séisme & 667941,2 \\
\hline Managua & Nicaragua & 1972 & séisme & 100001 \\
\hline Guatemala City & Guatemala & 1976 & séisme & 230001,2 \\
\hline Tangshan & Chine & 1976 & séisme & $242000^{1}$ \\
\hline Armero & Colombie & 1985 & $\begin{array}{l}\text { éruption volcanique } \\
\text { (lahars) }\end{array}$ & 250001 \\
\hline Mexico & Mexique & 1985 & séisme & 95001 \\
\hline San Salvador & Salvador & 1986 & séisme & 11001,3 \\
\hline Spitak & Arménie & 1988 & séisme & 250004 \\
\hline Manjil & Iran & 1990 & séisme & 400001,2 \\
\hline Kobé & Japon & 1995 & séisme & 53001 \\
\hline Tegucigalpa & Honduras & 1998 & cyclone & 3005 \\
\hline Izmit & Turquie & 1999 & séisme & 170001,2 \\
\hline Bhuj & Inde & 2001 & séisme & 150001,6 \\
\hline San Salvador & Salvador & 2001 & deux séismes & 11591 \\
\hline Bam & Iran & 2003 & séisme & 260001,2 \\
\hline Banda Aceh & Indonésie & 2004 & séisme et tsunami & 310007 \\
\hline Muzaffarabad & Pakistan & 2005 & séisme & $\begin{array}{c}\text { plus de } \\
100008\end{array}$ \\
\hline Bombay & Inde & 2005 & $\begin{array}{l}\text { inondations et } \\
\text { glissements de terrain }\end{array}$ & un millier 9 \\
\hline Bogalay & Birmanie & 2008 & cyclone & 1000010 \\
\hline Mianyang & Chine & 2008 & séisme & 2200011 \\
\hline Port-au-Prince & Haïti & 2010 & séisme & 21200012 \\
\hline
\end{tabular}

Sources : 1. CRED (Centre for Research on the Epidemiology of Disasters) ; 2. La Documentation Française ; 3. DesInventar ; 4. NSSP (Armenian National Survey for Seismic Protection) ; 5. Diario de Centro América ; 6. Tribune de Genève ; 7. Khaleej Times ; 8. World Bank ; 9. EchoGéo ; 10. Le Monde ; 11. Website official of Mianyang, may 31, 2008; 12. Comité de Defense Civile du Gouvernement de Haïti (estimation au 6 février 2010)

d'une partie du glacier du Huascarán. Plus récemment, le 15 août 2007, plusieurs petites villes, dont celles de Pisco et de Chincha Alta ont été touchées par un autre séisme, faisant plusieurs centaines de victimes et détruisant des dizaines de milliers de logements. Déjà en 1687, Pisco avait dû être déplacée suite à un tremblement de terre (Musset, 2002). 
Pour leur part, les villes boliviennes n'ont jamais enregistré de catastrophes de grande magnitude, du moins en termes de vies humaines. Ce constat est en partie lié à une population urbaine peu importante en Bolivie, jusqu'à ce que le gouvernement ne décide de développer l'axe urbain La Paz-CochabambaSanta Cruz5. Avec 69 morts et de très importants dégâts matériels, les inondations survenues à La Paz le 19 février 2002, peuvent être considérées comme l'une des plus grandes catastrophes urbaines connues en Bolivie.

Ces grandes catastrophes sont ainsi quasi inexistantes en Bolivie et relativement peu fréquentes, à l'échelle humaine, au Pérou et en Équateur. En revanche, les villes des trois pays andins ont enregistré une multitude d'évènements de moindre ampleur, liés à des phénomènes tels que des inondations, des glissements de terrain, des accidents technologiques, des incendies ou des pollutions accidentelles. Le cumul de ces dommages est très significatif sur le plan humain et matériel, pénalisant le développement économique et social de ces villes. A Lima, par exemple, environ 3000 évènements de ce type ont été enregistrés depuis 1970, soit 80 évènements par an, et ce sont les quartiers les plus vulnérables, les plus marginalisés, qui sont les plus touchés.

\section{LE PROGRAMME PACIVUR}

Dans ce contexte, le programme de recherche Pacivur (programme andin de formation et de recherche sur la vulnérabilité et les risques en milieu urbain) a été lancé en avril 2006 dans le cadre d'une convention signée entre l'IRD (Institut de Recherche pour le Développement) et la CAN (Communauté Andine). Un accord de collaboration a également été établi avec I'IFEA (Institut Français d'Études Andines, UMIFRE 17, CNRS-MAEE) et avec le programme européen Predecan (Prévention des Désastres dans la Communauté Andine) d'appui institutionnel à la CAN. Des collaborations étroites, parfois renforcées par des conventions6, ont été nouées avec les municipalités de La Paz, Lima et Quito, avec des universités7, les instituts de protection civile, des ONG comme l'ONG italienne Coopis et des institutions internationales comme Echo'.

Pacivur fait suite au programme de recherche "Système d'information et risques dans le District Métropolitain de Quito » développé dans le cadre d'une collaboration entre I'IRD et la municipalité de Quito entre 1999 et 2004, et constituant une première initiative de recherche de l'IRD sur les vulnérabilités

5 Cela a démarré au milieu des années 1950 avec une accélération depuis 1990.

6 Conventions avec la municipalité (Gobierno Municipal) de La Paz, I'IMP (Instituto Metropolitano de Planificación de Lima) et I'INDECI (Instituto Nacional de Defensa Civil del Perú).

7 Parmi ces universités : la Universidad Nacional Mayor de San Marcos, Lima (UNMSM); la Pontificia Universidad Católica del Ecuador, Quito (PUCE); la Universidad Mayor de San Andrés, La Paz (UMSA).

8 Cooperazione Internazionale (http://www.coopi.org/).

9 European Commission Humanitarian aid Office (Office d'aide humanitaire de la Commission Européenne). 
urbaines en Amérique du Sud, centrée sur la capitale équatorienne. L'approche originale de cette recherche (fondée sur les enjeux territoriaux de la ville), les nombreuses méthodologies d'analyse de la vulnérabilité mises au point et les résultats obtenus pour la municipalité de Quito ont été à l'origine du succès de ce premier programme. C'est pourquoi son extension à l'échelle régionale a été envisagée. Pacivur s'intéresse à trois pays andins : la Bolivie, le Pérou et l'Équateur, pays où I'IRD dispose d'une représentation permanente10.

Le programme Pacivur présente trois objectifs complémentaires : la production de connaissances utiles pour la gestion et la réduction des risques, en particulier dans les domaines de la vulnérabilité sociale, politique et territoriale ; la divulgation dans la région andine des résultats des travaux scientifiques, ce qui est précisément le but de ce numéro thématique ; la création d'un potentiel régional de recherche et de réflexion sur la vulnérabilité et les risques en milieu urbain par la formation de jeunes chercheurs andins et français. La recherche développée dans le cadre du programme Pacivur, à travers différents projets de recherche, s'articule autour de deux axes. Elle vise d'abord à comprendre les modalités de construction de la vulnérabilité dans les villes des pays andins, à caractériser et évaluer cette vulnérabilité et à apprécier sa portée en termes de risques et de conséquences humaines et matérielles. Elle a ensuite pour propos d'analyser les modalités de réduction de cette vulnérabilité à la fois dans un sens préventif (planification préventive urbaine) et dans une perspective de gestion des crises.

\section{OBJECTIFS ET ORGANISATION DU NUMÉRO THÉMATIQUE}

Ce numéro souhaite présenter les principaux résultats des travaux scientifiques réalisés sur la vulnérabilité urbaine dans les trois pays andins, produits par l'équipe Pacivur et par quelques chercheurs associés, depuis le lancement du programme en avril 2006. Au total, ce sont 20 personnes qui ont contribué à la réalisation de ce volume, pour la plupart des géographes11. Il porte essentiellement sur les trois capitales — La Paz, Lima et Quito— sur lesquelles se sont concentrées les activités scientifiques12.

Comme nous l'avons souligné précédemment, Pacivur est un programme de recherche, mais également un programme de formation de jeunes chercheurs andins et français sur le thème de la vulnérabilité urbaine. C'est pourquoi, si ce numéro thématique a pour premier objectif de restituer les résultats des recherches réalisées durant trois ans, il est en même temps une contribution à la formation de jeunes chercheurs dont certains n'avaient encore jamais publié d'articles

10 Sites internet : http://www.bo.ird.fr/ (Bolivie) ; http://www.ec.ird.fr/ (Équateur) ; http://www.peru. ird.fr/ (Pérou).

11 Un sociologue, une architecte, une cartographe et un informaticien ont également participé à l'élaboration et la rédaction de ce numéro thématique.

12 À l'exception de la province de Chincha, au Pérou, où des travaux de recherche ont été réalisés sur le processus de reconstruction qui a suivi le séisme de 2007. 
scientifiques. Ce numéro thématique a ainsi bénéficié du concours de plusieurs chercheurs expérimentés tout en constituant une plateforme de publication pour les plus jeunes.

Ce volume est organisé en quatre parties, qui constituent autant d'approches pertinentes et complémentaires de la vulnérabilité urbaine dans les pays andins.

La première partie questionne des évènements passés pour en tirer des enseignements en matière de vulnérabilité : vulnérabilité « de fond » qui résulte de processus socio-historiques sur le temps long, vulnérabilité « de circonstance» liée au contexte du moment et aux caractéristiques de la gestion de crise. Dans un premier temps, c'est une analyse statistique des accidents et des catastrophes survenus dans les trois capitales qui est proposée avec l'article de R. D'Ercole, S. Hardy et J. Robert. Cette présentation générale permet de mieux connaître les conditions de leur vulnérabilité. Elle amène en même temps à réfléchir aux informations disponibles pour ce type d'exercice et à l'insuffisance des bases de données existantes. Dans un deuxième temps, il s'agit de s'extraire de l'approche statistique et cartographique, pour pénétrer au cœur de chaque évènement afin de mieux comprendre, au-delà du phénomène physique, sa construction sociale et sa portée à la fois territoriale et institutionnelle. Sept études de cas sont présentées couvrant une gamme étendue de situations (lieux, types de phénomènes, extension des effets territoriaux, ancienneté de l'évènement, etc.). Deux évènements concernent Lima : le district de Lurigancho-Chosica affecté par des coulées torrentielles en 1987 (C. Abad) et la Margen Izquierda du Río Rímac (Cercado de Lima) affectée par le séisme du 15 août 2007 (J. Robert et R. D'Ercole). Deux autres études de cas sont proposées par S. Hardy à propos de La Paz. La première est la crise provoquée par les inondations du 19 février 2002 ; la deuxième est celle qui, en 2008, a fait suite à l'interruption de l'approvisionnement en eau d'une grande partie de la capitale bolivienne du fait de l'endommagement d'une canalisation. Les trois autres études de cas ont Quito pour scénario. Elles retracent et analysent les situations de crise déclenchées par I'activité du volcan Guagua Pichincha en 1998-1999 (R. D'Ercole, P. Metzger et A. Sierra), un grand incendie survenu en 2003 dans le quartier Santa Rosa de Chillogallo (J. Estacio) et un effondrement de chaussée en 2008 ayant concerné Le Trebol, lieu stratégique de la mobilité à Quito (D. Salazar, F. Demoraes, N. Bermúdez et $\mathrm{S}$. Zavgorodniaya). Ces études de cas ont été réalisées en suivant une méthodologie commune, ce qui permet la comparaison. Une synthèse est enfin proposée dans l'objectif de tirer des enseignements plus généraux en matière de conséquences, de facteurs de vulnérabilité et d'incidence des évènements analysés (texte de R. D'Ercole, S. Hardy et J. Robert).

La deuxième partie de ce numéro thématique s'interroge sur les processus passés et actuels de construction et de consolidation de la vulnérabilité. À travers l'analyse de deux secteurs de Lima (Margen Izquierda del Río Rímac — Cercado de Lima — et El Agustino), J. Robert et A. Sierra montrent comment les représentations du risque, le cadre institutionnel et les modes de gouvernement des territoires urbains sont au cœur de la construction de la vulnérabilité. En même temps, l'absence d'une politique publique concertée de prévention des risques contribue à consolider cette vulnérabilité. Les mécanismes de renforcement de la vulnérabilité apparaissent 
également dans l'étude de cas concernant la province de Chincha, très durement touchée par le séisme du 15 août 2007. À partir d'une enquête menée auprès de 1703 familles affectées par cet séisme, I'article de R. D'Ercole, R. Cavagnoud, M. Morel et $\mathrm{P}$. Vernier souligne I'augmentation de la vulnérabilité et la dégradation des conditions de vie de milliers de familles. Ce renforcement de la vulnérabilité des plus pauvres s'explique notamment par une politique d'aide à la reconstruction insuffisante, inadaptée et inégalitaire. Pour sa part, I'article de J. Estacio montre, à travers une approche historique, sociale et territoriale, comment se construit et se transforme le risque technologique autour du Beaterio, principal centre de stockage de combustibles de Quito. Il analyse les conséquences des options prises par les pouvoirs publics pour réduire le risque, ces choix portant essentiellement sur le contrôle de l'aléa et non pas sur celui de l'urbanisation. La manière dont se construit la vulnérabilité de Lima est abordée sous I'angle des déchets solides par M. Durand et P. Metzger. Ces derniers proposent une lecture de la production des espaces par la gestion des déchets. Ils expliquent comment cette gestion opère un transfert de vulnérabilité des espaces producteurs vers d'autres lieux, pauvres et marginalisés, dont le rôle dans le traitement des déchets est fondamental compte tenu des insuffisances des pouvoirs publics dans ce domaine. Le dernier article, proposé par J. Robert, T. Serrano, R. D’Ercole et P. Pigeon montre comment la complexité associée à l'incertitude produit de la vulnérabilité à partir du cas du Valle de Los Chillos (Quito, Équateur) exposé aux éruptions du volcan Cotopaxi. II souligne le fait que réduire le risque nécessite d'agir dans l'incertitude et qu'il est possible de réduire cette incertitude en démêlant la complexité. Des travaux de recherche géographiques allant dans ce sens sont ainsi présentés.

La troisième partie aborde la question de la réduction des vulnérabilités sous l'angle des politiques urbaines de gestion des risques menées dans les trois capitales. En premier lieu, I'article de S. Hardy s'intéresse à l'évolution de ces politiques à La Paz, dans le temps et dans l'espace : I'accent a successivement été placé sur la planification urbaine, puis sur la construction d'ouvrages de protection et actuellement sur la gestion des situations de crise, affichant par là même les limites des politiques de prévention. À Quito, la politique de lutte contre le risque repose aussi sur des logiques planificatrices qui se sont développées sur le temps long. Dans ce contexte, I'article de A. Sierra décrit l'évolution des aménagements réalisés sur les versants du volcan Pichincha, marquée par une prise en compte de plus en plus explicite de la vulnérabilité sociale, tout en explorant les arguments qui guident ces aménagements. Ce sont principalement des actions ponctuelles de gestion des risques qui permettent de rendre compte de la politique de prévention des risques dans le cas de Lima présenté par A. Sierra, J. Robert, M. Durand et C. Abad. Ces expériences ponctuelles, sur le plan spatial comme sur le plan sectoriel, traduisent une lacune de fond : I'absence d'une politique métropolitaine de gestion des risques à Lima. L'article de S. Hardy et E. Combaz considère l'implication croissante des autorités publiques boliviennes dans le domaine de la gestion de crise. II s'intéresse plus spécifiquement aux problèmes du relogement de populations sinistrées à La Paz dans un contexte de pénurie de terrains urbanisables exempts de risques. 
La quatrième partie, composée de cinq articles, considère la réduction des vulnérabilités urbaines à partir de l'apport des chercheurs en termes d'approches scientifiques, de concepts, de méthodes et d'outils. L'analyse de vulnérabilité des réseaux routiers urbains constitue une approche très pertinente pour comprendre la vulnérabilité territoriale des grandes villes. C'est ce que démontrent J. Nuñez et F. Demoraes à partir du cas de La Paz et secondairement de Quito (encart). Selon D. Salazar et R. D'Ercole, s'appuyant sur des recherches menées dans le Valle de Los Chillos (Quito), les études de perception du risque permettent de relier de manière constructive les points de vue des différents acteurs potentiellement impliqués par une crise volcanique (population, scientifiques, autorités). Elles sont donc très utiles en termes de préparation à la gestion de crise. L'article de S. Hardy porte sur l'évaluation et la gestion de la vulnérabilité des districts 5 et 6 de la municipalité d'El Alto. II représente un bon exemple de recherche appliquée, venue en appui d'un projet de préparation aux catastrophes financé par la Commission Européenne et géré par l'ONG Coopi. L'intérêt d'une problématique de recherche géographique s'interrogeant sur les dimensions spatiales de la gestion des crises est démontré dans I'article de R. D'Ercole et P. Metzger. Les auteurs exposent les apports d'une recherche portant sur l'articulation spatiale entre espaces vulnérables et espaces ressources de la gestion de crise et proposent une première application sur le cas des établissements de santé de Quito. Le dernier article (P. Metzger et R. D’Ercole) met davantage l'accent sur les concepts qui orientent les approches scientifiques. À partir de différents exemples et travaux réalisés sur les vulnérabilités et crises urbaines, il propose une première réflexion théorique sur la notion de transmission de vulnérabilité, essentielle pour comprendre et gérer les risques encourus au sein des territoires urbains.

\section{Références citées}

ALBOUY, F.-X., 2002 - Le temps des catastrophes, 172 pp. ; Paris : Descartes \& Cie.

BLAIKIE, P., CANNON, T., DAVIS, I. \& WISNER, B., 1994 - At risk. Natural people's vulnerability and disasters, 280 pp. ; New-York : Routledge.

CHESTER, D. K., 2002 - Overview: hazard and risk. In : Applied Geomorphology: theory and practice (R. J. Allison, ed): 251-264; Chichester : John Willey and Sons.

D'ERCOLE, R., 1994 - Les vulnérabilités des sociétés et des espaces urbanisés : concepts, typologie, modes d'analyse. Revue de Géographie Alpine, $\mathbf{n}^{\circ} \mathbf{4}, \mathbf{T}$. LXXXII : 87-96. Numéro thématique : "Croissance urbaine et risques naturels dans les montagnes des pays en développement ».

D'ERCOLE, R. \& THOURET, J.-C., 1995 - Croissance urbaine et risques naturels : présentation introductive. Bulletin de l'Association de Géographes Français, $\mathbf{n}^{\circ} \mathbf{4}$ : 311-338. Numéro thématique : «Croissance urbaine et risques naturels (en particulier dans les pays en développement) ».

D'ERCOLE, R., GLUSKI, P., HARDY, S. \& SIERRA, A., 2009 - Vulnérabilités urbaines dans les pays du Sud. Présentation du dossier. Cybergeo : European Journal of Geography. [En ligne], Dossiers, Vulnérabilités urbaines au sud, URL : http://www.cybergeo.eu/ index22151.html 
DUBOIS-MAURY, J. \& CHALINE, C., 2002 - Les risques urbains 208 pp. ; Paris : Armand Colin.

LAVELL, A., 1999 - Gestión de riesgos ambientales urbanos, 15 pp.; Quito: FLACSO, La Red. http://www.desenredando.org/public/articulos/1999/grau/index.html.

MUSSET, A., 2002 - Villes nomades du nouveau monde, 398 pp. ; Paris : Ed. EHSS.

PELLING, M., 2003 - The vulnerability of cities: natural disasters and social resilience, 256 pp. ; Londres : Earthscan.

PIGEON, P., 1996 - La gestion des risques urbains. In: Risques naturels, risques de sociétés (A. Bailly ed.): 51-62 ; Paris : Economica.

PIGEON, P., 2007 - L'environnement au défi de I'urbanisation, 189 pp. ; Rennes : PUR.

UNFPA, 2007 - État de la population mondiale 2007. Libérer le potentiel de la croissance urbaine - http://www.unfpa.org/swp/2007/french/introduction.html

VIÉ-LE-SAGE, R., 1989 - La terre en otage. Gérer les risques naturels majeurs, 246 pp.; Paris : Seuil. 